\title{
ESTUDIO COMPARATIVO IN VITRO DE LA RESISTENCIA A LA COMPRESIÓN DE RESINAS PARA EL SECTOR POSTERIOR DE ACUERDO CON SU ESPESOR
}

\author{
${ }^{1}$ Andrea del Pilar Bayona Vallejo, ${ }^{1}$ Liliana Duarte Molina, ${ }^{1}$ Karol Yesenia Jiménez Camacho, ${ }^{2}$ Jorge Guillermo Díaz Rodríguez \\ ${ }^{1}$ Estudiante X semestre F. de Odontología Universidad Santo Tomás, Bucaramanga, Colombia. \\ ${ }^{2}$ Ingeniero Mecánico U. Industrial de Santander, Máster of Science Mechanical Engineering U. of North Texas, Docente Universidad Santo Tomás, Bucaramanga, Colombia.
}

Autor responsable de correspondencia: Jorge Guillermo Díaz Rodríguez.

Correo electrónico: jorgegdiaz@gmail.com

\begin{abstract}
RESUMEN
Objetivo: Comparar el comportamiento In vitro de la resinas de tipo condensable Filtek P60 (3M ESPE), Surefil (Dentsply), Tetric N-Ceram (Ivoclar Vivadent) al ser sometidas a fuerzas compresivas.

Materiales y métodos: Se realizó un estudio experimental In vitro con una muestra de cinco objetos de prueba desde $1 \mathrm{~mm}$ hasta $5 \mathrm{~mm}$ de espesor $\mathrm{x} 4 \mathrm{~mm}$ de diámetro. El total lo constituyeron 25 especímenes por cada resina condensable a evaluar. De esta manera, se evaluaron 75 objetos de prueba. Éstos fueron sometidos al test de compresión por medio de la máquina de ensayo universal Shimadzu Autograph AG-i 250.

Resultados: Se obtuvieron en promedio 228,9 \pm 78,7MPa, 210,6 \pm 74,9MPa y 190,7 \pm 71,7MPa de resistencia a la compresión para las resinas Filtek P60 (3M ESPE), Tetric N Ceram (Ivoclar Vivadent) y SureFil (Dentsply), respectivamente, sin diferencias estadísticamente significativas entre ellas. Por otro lado, se observó que, según la altura de la resina, la máxima resistencia medida fue 294,5 $\pm 71,7 \mathrm{MPa}$ para la de $1 \mathrm{~mm}$ y la más baja fue para la de $4 \mathrm{~mm}$ de altura con un promedio de 153,8 \pm 36,6MPa.

Conclusión: La resina de mayor resistencia a la compresión fue la Filtek P60 (3M ESPE) seguida de la Tetric N Ceram (Ivoclar Vivadent) y, por último, de la SureFil (Dentsply), aunque no se presentaron diferencias estadísticamente significativas. La probeta de mayor resistencia a la compresión con respecto al espesor fue la de $1 \mathrm{~mm}$, seguida por las de 3, 2, 5 y 4mm. [Bayona AP, Duarte L, Jiménez KY, Díaz JG. Estudio comparativo In vitro de la resistencia a la compresión de resinas para el sector posterior de acuerdo con su espesor. Ustasalud 2010; 9: 67 - 74]
\end{abstract}

Palabras clave: Materiales dentales, Resina compuesta, Resistencia a la compresión.

\section{IN VITRO COMPARATIVE STUDY OF COMPOSITES' COMPRESSIVE RESISTANCE FOR POSTERIOR SECTOR ACCORDING TO THEIR THICKNESS}

\begin{abstract}
Objective: To compare the In vitro behavior of packable composites Filtek P60 (3M ESPE), Surefil (Dentsply), Tetric N-Ceram (Ivoclar Vivadent), when subjected to compressive strength.

Methods: An experimental In vitro study was done in samples of $4 \mathrm{~mm}$ in diameter coupons ranging from $1 \mathrm{~mm}$ to $5 \mathrm{~mm}$ thick, for a total of 25 specimens for each evaluated packable resin. The sample size was 75 coupons. These were subjected to a compression test through a Shimadzu Autograph AG-i 250 universal testing machine.

Results: Obtained average compression strength values were: $228,9 \pm 78,7 \mathrm{MPa}, 210,6 \pm 74,9 \mathrm{MPa}$ and 190,7 \pm 71,7MPa for composites Filtek P60 (3M ESPE), Tetric N Ceram (Ivoclar Vivadent) y SureFil (Dentsply) respectively, without significant statistic differences between them. On the other hand, it was observed that, according to the resin thickness, the maximum resistance measured was $294,5 \pm$ 71,7MPa for the $1 \mathrm{~mm}$ high sample and the lowest was for $4 \mathrm{~mm}$ one with an average of 153,8 $\pm 36,6 \mathrm{MPa}$.

Conclusions: In the experimental tests the composite with the highest compression strength was Filtek P60 (3M ESPE) followed by Tetric N Ceram (Ivoclar Vivadent) and at last SureFil (Dentsply), although no significant sadistic differences were evident. The sample with the highest compression strength, according to the height, was the $1 \mathrm{~mm}$ one, followed by the $3,2,5$, and $4 \mathrm{~mm}$. ones
\end{abstract}

Key words: Dental materials, Composite resins, Compression strength. 


\section{INTRODUCCIÓN}

Cuando hay pérdida de la estructura dental debido a caries dental o trauma, es necesario realizar restauraciones con diferentes materiales, con el fin de reemplazar el tejido, recuperar su forma y función, y lograr que dichos materiales interactúen de manera adecuada con el tejido dental remanente. ${ }^{1}$

Las resinas se introdujeron en el mercado hacia los años 50 como material restaurador que reemplazaría a la amalgama, por ser estéticas y afines al diente. Las primeras resinas presentaron problemas en la adhesión por la alta contracción de polimerización que produjo filtraciones marginales elevadas en las restauraciones. Estos problemas se solucionaron con el tiempo al incorporar partículas de relleno que fortalecían la resina y disminuían el material de la matriz. ${ }^{2}$

Un factor importante al restaurar los dientes con resina compuesta, es la resistencia del material restaurador; esta propiedad mecánica del material permite que la restauración sirva para sus funciones de manera eficaz, segura y por un periodo razonable, es decir, por el mayor tiempo posible. En otras palabras, la resistencia se refiere a la tensión máxima que puede soportar un material antes de fracturarse. ${ }^{1}$

En odontología restaurativa es importante conocer el comportamiento de las resinas frente a las fuerzas a las que se ven sometidas durante su uso en boca. Bajo las cargas masticatorias, las propiedades mecánicas del material restaurativo juegan un rol primordial al resistir el esfuerzo, la fatiga, el desgaste y la fractura generados en este proceso. Esto es de especial relevancia para las restauraciones posteriores, debido a las fuerzas compresivas estáticas ejercidas en esta zona. ${ }^{3}$

El objetivo del presente estudio fue evaluar el comportamiento de tres resinas para el sector posterior (Filtek $^{\mathrm{TM}}$ P60 - 3M ${ }^{\mathrm{TM}}$ ESPE; SureFil ${ }^{\circledR}$ - Dentsply; Tetric ${ }^{\circledR}$ Ceram HB - Ivoclar Vivadent) al ser sometidas a fuerza de compresión y al hacer uso de una máquina universal de ensayos (Shimadzu Autograph AG-I 250).

\section{MATERIALES Y MÉTODOS}

Se realizó un estudio experimental In vitro. Se confeccionaron en resina 75 muestras con el fin de aplicar la prueba de resistencia a la compresión. Los materiales usados fueron Filtek P60 (3M ESPE), SureFil (Dentsply) y Tetric N-Ceram (Ivoclar Vivadent). Se seleccionaron cinco muestras de distintos espesores de cada una de las resinas, por consiguiente, 25 probetas se sometieron al test de compresión por cada resina evaluada.
El diámetro de cada muestra se confeccionó de acuerdo con las medidas de estudios realizados anteriormente. ${ }^{3,4}$ De esta manera, se obtuvieron especímenes de forma cilíndrica de $1 \mathrm{~mm}$ a $5 \mathrm{~mm}$ de altura y $4 \mathrm{~mm}$ de diámetro así:

- $1 \mathrm{~mm}$ de altura $\mathrm{x} 4 \mathrm{~mm}$ de diámetro.

- $2 \mathrm{~mm}$ de altura $\mathrm{x} 4 \mathrm{~mm}$ de diámetro.

- $3 \mathrm{~mm}$ de altura $x 4 \mathrm{~mm}$ de diámetro.

- $4 \mathrm{~mm}$ de altura $\mathrm{x} 4 \mathrm{~mm}$ de diámetro.

- $5 \mathrm{~mm}$ de altura $\mathrm{x} 4 \mathrm{~mm}$ de diámetro.

Para garantizar la uniformidad de las muestras se fabricaron cinco moldes en acero inoxidable, uno para cada espesor (Figura 1). Los especímenes se prepararon por la técnica incremental, este incremento fue de $2 \mathrm{~mm}$, posteriormente se fotocuraron de acuerdo con las instrucciones del fabricante, ${ }^{4,5}$ con una lámpara LED (DEMI - Kerr) (Figura 2). Después de fotocurar, cada tipo de material se colocó en un recipiente y se almacenaron en agua destilada a temperatura ambiente durante 24 horas. ${ }^{6}$ La elaboración de las muestras se llevó a cabo por un solo operador para evitar el error sistemático que se podría generar producto de la subjetividad de los preparadores.

Se colocaron en la máquina una a una cada muestra y se sometieron a la prueba de compresión. El ensayo de compresión consistió en aplicar a la probeta, en la dirección de su eje longitudinal, una carga estática que provoca un acortamiento de la misma y cuyo valor se incrementa hasta la rotura o suspensión del ensayo. ${ }^{7}$ Para obtener la resistencia máxima de cada objeto de prueba, se sometió a una fuerza progresivamente en aumento hasta su ruptura mediante una máquina universal de ensayos Shimadzu Autograph AG-i $250 \mathrm{kN}$ (Figura 3). ${ }^{8}$ La velocidad de la carga aplicada fue de $0.05 \mathrm{~mm} / \mathrm{min} .{ }^{4}$

Para las pruebas se usó la celda de carga de $100 \mathrm{kN}$ disponible en el laboratorio de caracterización de materiales de ingeniería mecatrónica de la Universidad Santo Tomás, Bucaramanga. Durante todas las pruebas la celda tuvo calibración vigente.

\section{Preparación de la muestra}

A cada molde se le aplicó una capa de agente separador. Se colocó el molde sobre una banda de película transparente sobre una lámina de vidrio para microscopio. Se llenó el molde con la resina, preparado de acuerdo con las instrucciones del fabricante, con el cuidado de no permitir la formación de burbujas de aire como se ve en la Figura 4. Se sobrellenó ligeramente el molde y se puso una segunda película transparente en la cubierta, seguido de la segunda lámina de microscopio. 


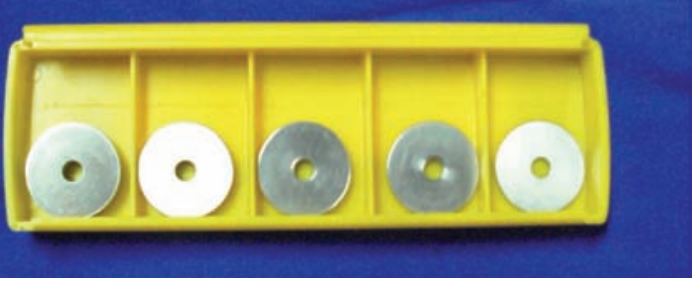

Figura 1. Moldes de acero inoxidable.

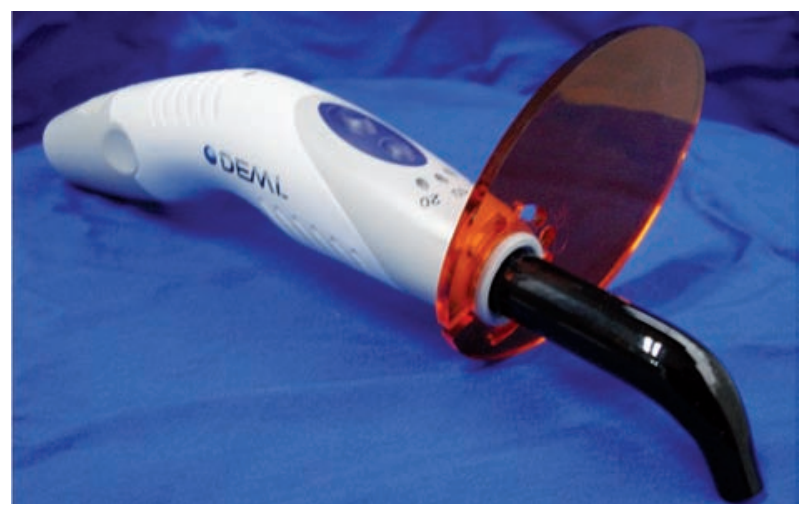

Figura 2. Lámpara de fotocurado DEMI - Kerr.

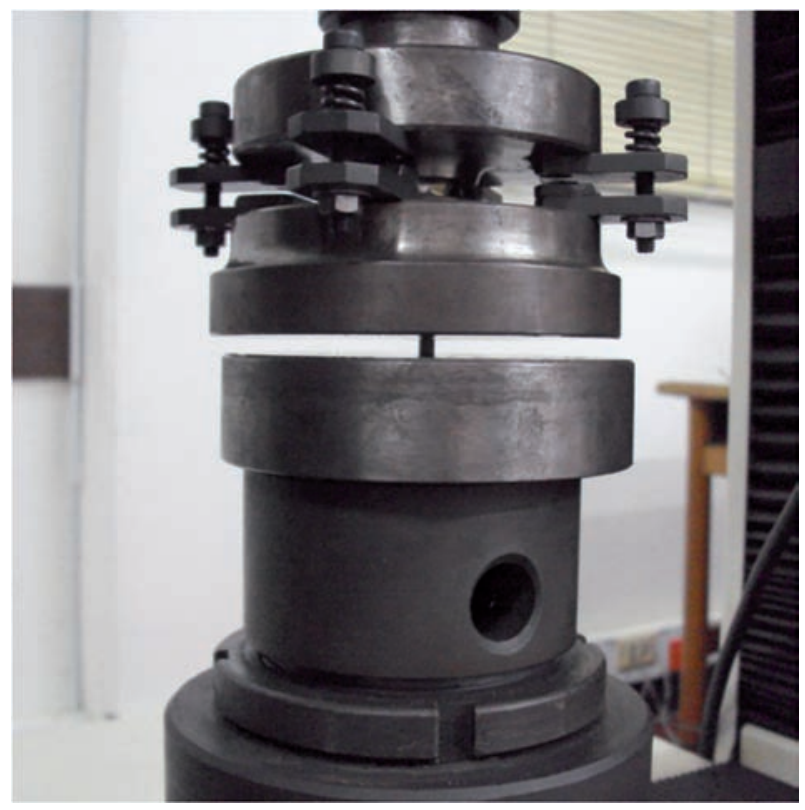

Figura 3. Test de compresión en la máquina universal de ensayos.

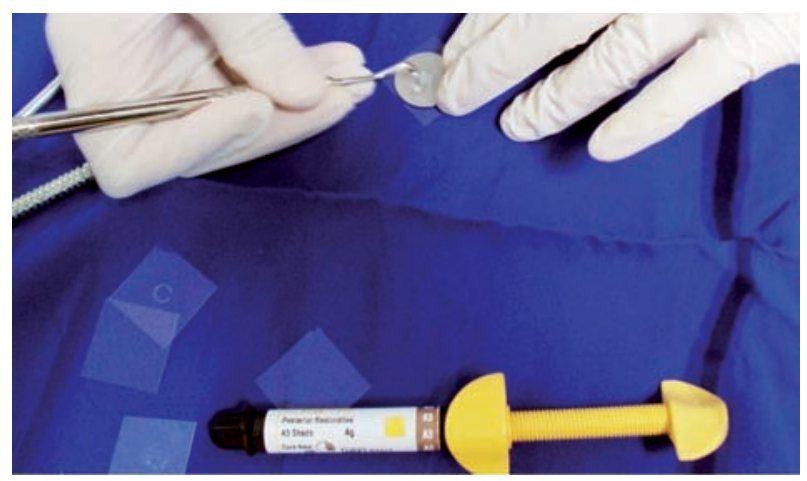

Figura 4. Elaboración de la muestra.

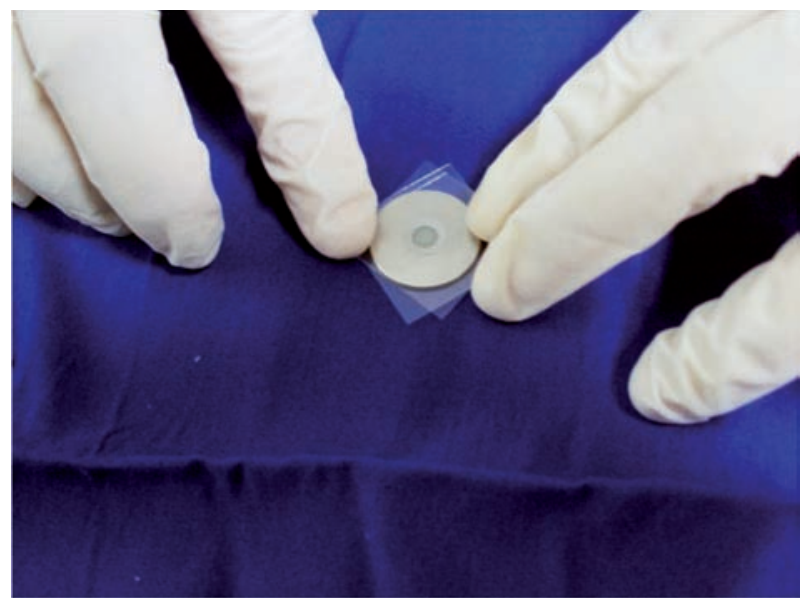

Figura 5. Elaboración de la muestra.

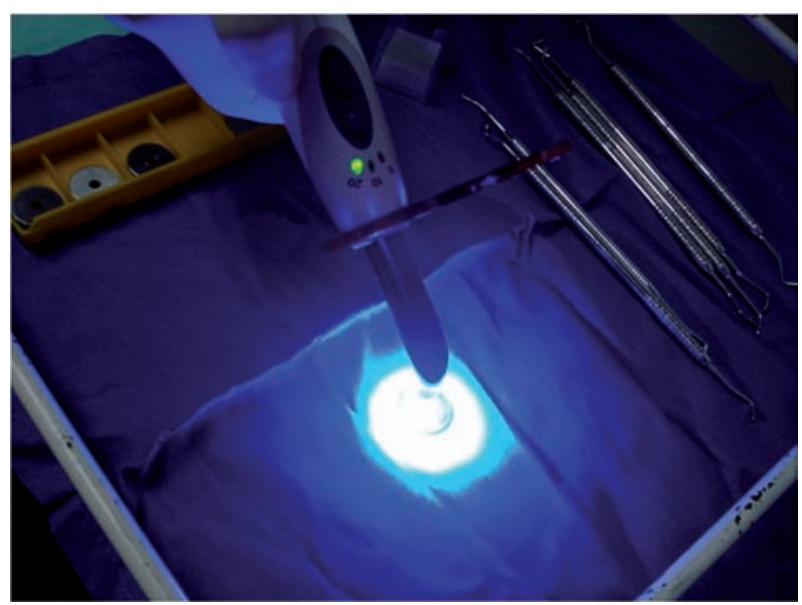

Figura 6. Fotopolimerización de la muestra.

Se presionó el molde y las tiras de película entre las dos láminas de vidrio para eliminar el exceso de material (Figura 5). Se retiró la lámina de microscopio que cubría la tira superior de película y se colocó cuidadosamente la ventana de salida de la fuente de energía externa contra la tira de película (Figura 6). Se fotocuró el material durante el tiempo recomendado por el fabricante. ${ }^{9}$

\section{Categorización de las fallas}

Para la estimación de la falla, el software Trapezium 2,23 le puede estipular una acción a la máquina de acuerdo con la rata de aumento de la razón de carga. Para esto se coloca un valor que indique que hubo ruptura. Como en este caso se desconocían datos para establecer un cambio de rata que pueda traducirse en una falla, las fallas se evaluaron visual y aditivamente, de acuerdo con algún salto en la curva que arrojara el software como se demuestra en una curva característica expuesta en la Figura 7. Todas las fallas se presentaron en sentido axial a la muestra. En la Figura 8, se observa una grieta en una de las probetas después de ser sometida al ensayo. 


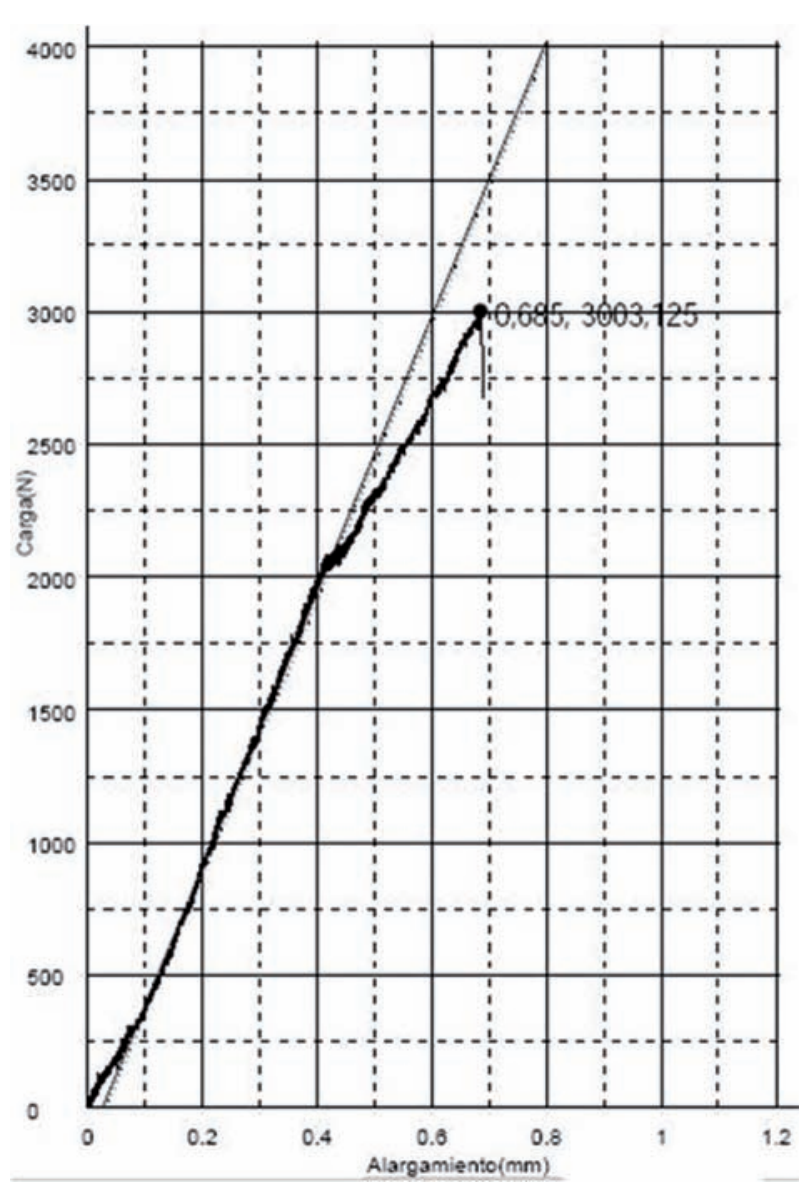

Figura 7. Curva típica carga vs alargamiento para una muestra.

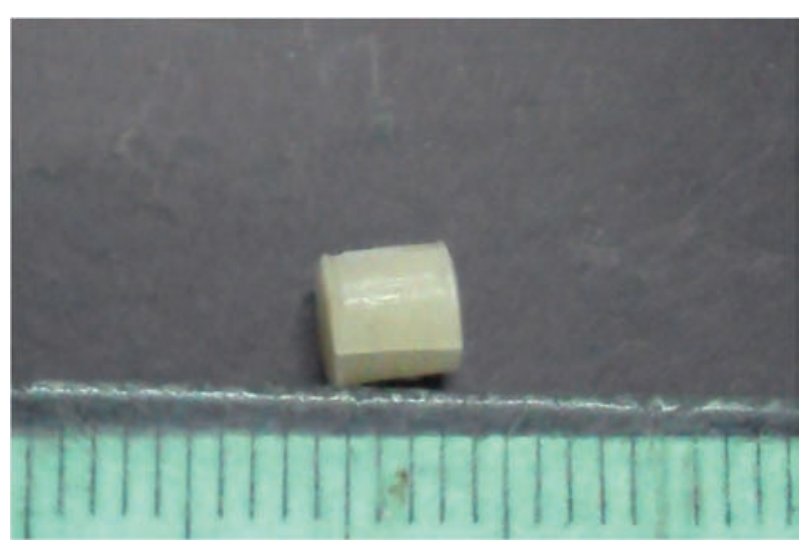

Figura 8. Probeta después de ser sometida al ensayo de compresión.

\section{Análisis estadístico}

Todos los datos obtenidos de la prueba fueron tabulados y analizados estadísticamente. Para el análisis bivariado se utilizó ANOVA de una vía y la prueba de Bonferroni. ${ }^{8}$ En el análisis multivariado se aplicó una regresión lineal múltiple, la resistencia a la compresión fue la variable dependiente, la marca y el espesor fueron las variables explicatorias para analizar su efecto conjunto sobre la resistencia.

\section{RESULTADOS}

La muestra estuvo conformada por 75 objetos de prueba de 5 espesores diferentes (desde $1 \mathrm{~mm}$ hasta $5 \mathrm{~mm}$ ) y tres tipos de resina (Filtek P60 (3M ESPE), SureFil (Dentsply), Tetric N-Ceram (Ivoclar Vivadent)), los cuales fueron sometidos a la prueba de compresión.

Durante el estudio, se obtuvieron en promedio 228,9 $\pm 78,7 \mathrm{MPa}, 210,6 \pm 74,9 \mathrm{MPa}$ y $190,7 \pm 71,7 \mathrm{MPa}$ de resistencia a la compresión para las marcas Filtek P60 (3M ESPE), Tetric N Ceram (Ivoclar Vivadent) y SureFil (Dentsply), respectivamente, sin diferencias estadísticamente significativas entre ellas. Por otro lado, se observó que según el espesor de resina, la máxima fuerza aplicada fue 294,5 \pm 71,7MPa para $1 \mathrm{~mm}$ y la resistencia más baja fue para los $4 \mathrm{~mm}$ de espesor con un promedio de $153,8 \pm 36,6 \mathrm{MPa}(\mathrm{p}=0,000)$.

Al comparar las marcas por espesor (Tabla 1, Gráfico 1), no hubo diferencias estadísticamente significativas entre marcas, salvo en el espesor de $3 \mathrm{~mm}$. En la Tabla 2 se presentan las diferencias entre marcas para cada espesor, se confirma que no hubo diferencias estadísticamente significativas entre ellas para los espesores de $1 \mathrm{~mm}, 2 \mathrm{~mm}, 4 \mathrm{~mm}$ y $5 \mathrm{~mm}$. Mientras que, en el espesor de $3 \mathrm{~mm}$ la marca SureFil, toleró 68,4MPa menos comparado con la marca P60 $(\mathrm{p}=0,044)$ y $66,84 \mathrm{MPa}$ menos comparado con la marca Tetric N Ceram ( $p=0,05)$.

Igualmente, se comparó el espesor según marca. En la marca Filtek P60 (3M ESPE) (Tabla 3), el espesor de $1 \mathrm{~mm}$ fue estadísticamente diferente del espesor de $2 \mathrm{~mm}, 4 \mathrm{~mm}$ y $5 \mathrm{~mm}$, con diferencias de $-152,5,-155,3$ y $-121,8 \mathrm{Mpa}$, respectivamente; fue siendo mayor la fuerza soportada por el espesor de $1 \mathrm{~mm}$.

En la marca Tetric N Ceram (Ivoclar Vivadent), el espesor de $1 \mathrm{~mm}$ soportó mayor resistencia que los espesores de 2, 4 y 5mm; así como el espesor de $3 \mathrm{~mm}$ fue más resistente que el espesor de $5 \mathrm{~mm}$, en todos los casos (Tabla 4).

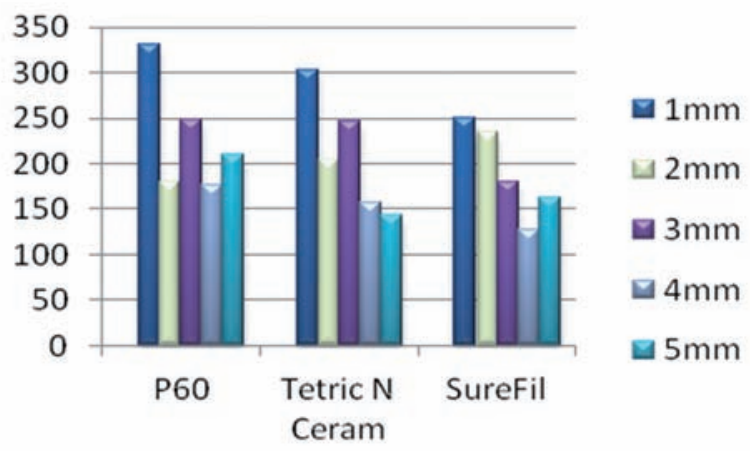

Gráfico 1. Promedios de resistencia al test de compresión (MPa), según marca y espesor del material. 


\section{ARTÍCULO ORIGINAL}

En la marca SureFil (Dentsply) se encontraron diferencias estadísticamente significativas entre el espesor $1 \mathrm{~mm}$ y $4 \mathrm{~mm}$, siendo mayor la fuerza soportada por el espesor de $1 \mathrm{~mm}(\mathrm{p}=0,046)$.

Se analizó el efecto conjunto de la marca y el espesor de la resina empleada sobre la fuerza compresiva soportada, mediante una regresión lineal múltiple que incluyó como variable dependiente la resistencia a la compresión y como variables explicatorias la marca y el espesor. Se encontró que este modelo explicó el $47 \%$ de la variabilidad de la resistencia del material, la cual disminuye con las marcas SureFil (Dentsply) y Tetric N Ceram (Ivoclar Vivadent); así como con los espesores de 2, 3, 4 y 5mm (Tabla 6).

Tabla 1. Resistencia a la compresión (MPa) según espesor y marca de resina.

\begin{tabular}{ccccc}
\hline \multirow{2}{*}{ Espesor } & P60 & Tetric N Ceram & SureFil & p \\
& $\mathbf{x} \pm \mathbf{D E}$ & $\mathbf{x} \pm \mathbf{D E}$ & $\mathbf{x} \pm \mathbf{D E}$ & 0,191 \\
$1 \mathrm{~mm}$ & $331,4 \pm 43,7$ & $302,7 \pm 57,8$ & $249,3 \pm 91,7$ & 0,237 \\
$2 \mathrm{~mm}$ & $178,9 \pm 5,4$ & $203,6 \pm 44,3$ & $233,8 \pm 70,5$ & $0,023^{*}$ \\
$3 \mathrm{~mm}$ & $248,4 \pm 33,9$ & $246,9 \pm 29,5$ & $180,0 \pm 48,1$ & 0,102 \\
$4 \mathrm{~mm}$ & $176,1 \pm 21,5$ & $157,4 \pm 46,4$ & $127,8 \pm 24,2$ & 0,407 \\
$5 \mathrm{~mm}$ & $209,6 \pm 113,6$ & $142,3 \pm 60,5$ & $162,7 \pm 42,9$ & \\
\hline
\end{tabular}

Tabla 2. Diferencias entre marcas de resina en la resistencia a la compresión (MPa) en el espesor de 1mm, $2 \mathrm{~mm}, 3 \mathrm{~mm}, 4 \mathrm{~mm}$ y $5 \mathrm{~mm}$.

\begin{tabular}{|c|c|c|c|c|c|}
\hline \multirow{2}{*}{ Marca } & \multirow{2}{*}{ Espesor } & P60 & \multirow{2}{*}{ p } & Tetric N Ceram & \multirow{2}{*}{ p } \\
\hline & & Diferencia $\mathrm{x}$ & & Diferencia $x$ & \\
\hline Tetric N Ceram & \multirow{2}{*}{$1 \mathrm{~mm}$} & $-28,7$ & 1.000 & & \\
\hline SureFil & & $-82,1$ & 0,235 & $-53,4$ & 0,70 \\
\hline Tetric N Ceram & \multirow{2}{*}{$2 \mathrm{~mm}$} & $-24,8$ & 1.000 & & \\
\hline SureFil & & 54,9 & 0,290 & 30,2 & 1,00 \\
\hline Tetric N Ceram & \multirow{2}{*}{$3 \mathrm{~mm}$} & $-1,5$ & 1.000 & & \\
\hline SureFil & & $-68,4$ & $0,044^{*}$ & $-66,8$ & $0,05^{*}$ \\
\hline Tetric N Ceram & \multirow{2}{*}{$4 \mathrm{~mm}$} & $-18,7$ & 1.000 & & \\
\hline SureFil & & $-48,4$ & 0,112 & $-29,5$ & 0,533 \\
\hline Tetric N Ceram & \multirow{2}{*}{$5 \mathrm{~mm}$} & $-67,2$ & 0,599 & & \\
\hline SureFil & & $-46,8$ & 1,000 & 20,4 & 1,000 \\
\hline
\end{tabular}

${ }^{*} \mathrm{p}<0,05$.

Tabla 3. Diferencias entre espesor de la resina en la resistencia a la compresión (MPa) en la marca P60.

\begin{tabular}{ccccccccc}
\hline Espesor & $\begin{array}{c}1 \mathrm{~mm} \\
\text { Diferencia } \\
\text { de } \mathbf{x}\end{array}$ & $\mathbf{p}$ & $\begin{array}{c}\text { 2mm } \\
\text { Diferencia } \\
\text { de } \mathbf{x}\end{array}$ & $\mathbf{p}$ & $\begin{array}{c}3 \mathrm{~mm} \\
\text { Diferencia } \\
\text { de } \mathbf{x}\end{array}$ & $\mathbf{p}$ & $\begin{array}{c}4 \mathrm{~mm} \\
\text { Diferencia } \\
\text { de } \mathbf{x}\end{array}$ & $\mathbf{p}$ \\
\hline $2 \mathrm{~mm}$ & $-152,5$ & $0,004^{*}$ & & & & & & \\
$3 \mathrm{~mm}$ & $-82,9$ & 0,333 & 69,5 & 0,697 & & & & \\
$4 \mathrm{~mm}$ & $-155,3$ & $0,004^{*}$ & $-2,8$ & 1,000 & $-72,3$ & 0,601 & & 1,000 \\
$5 \mathrm{~mm}$ & $-121,8$ & $0,031^{*}$ & 30,7 & 1,000 & $-38,8$ & 1,000 & 33,5 & \\
\hline
\end{tabular}

${ }^{*} \mathrm{p}<0,05$.

Tabla 4. Diferencias entre espesor de la resina en la resistencia a la compresión (MPa) en la marca Tetric N Ceram.

\begin{tabular}{cccccccc}
\hline Espesor & $\begin{array}{c}\text { 1mm } \\
\text { Diferencia } \\
\text { de } \mathbf{x}\end{array}$ & $\mathbf{p}$ & $\begin{array}{c}\text { 2mm } \\
\text { Diferencia } \\
\text { de } \mathbf{x}\end{array}$ & $\mathbf{p}$ & $\begin{array}{c}\text { 3mm } \\
\text { Diferencia } \\
\text { de } \mathbf{x}\end{array}$ & $\mathbf{p}$ & $\begin{array}{c}\mathbf{4 m m} \\
\text { Diferencia } \\
\text { de } \mathbf{x}\end{array}$ \\
\hline $2 \mathrm{~mm}$ & $-99,0$ & $0,045^{*}$ & & & & & \\
$3 \mathrm{~mm}$ & $-55,8$ & 0,867 & 43,2 & 1,000 & & & \\
$4 \mathrm{~mm}$ & $-145,3$ & $0,001^{*}$ & $-46,3$ & 1,000 & $-89,5$ & 0,090 & $-15,02$ \\
$5 \mathrm{~mm}$ & $-160,3$ & $0,000^{*}$ & $-61,3$ & 0,617 & $-104,5$ & $0,030^{*}$ & 1,000 \\
\hline
\end{tabular}

${ }^{*} \mathrm{p}<0,05$. 
ARTÍCULO ORIGINAL

Tabla 5. Diferencias entre espesor de la resina en la resistencia a la compresión (MPa) en la marca SureFil.

\begin{tabular}{ccccccccc}
\hline Espesor & $\begin{array}{c}\text { 1mm } \\
\text { Diferencia } \\
\text { de } \mathbf{x}\end{array}$ & $\mathbf{p}$ & $\begin{array}{c}\text { 2mm } \\
\text { Diferencia } \\
\text { de } \mathbf{x}\end{array}$ & $\mathbf{p}$ & $\begin{array}{c}\text { 3mm } \\
\text { Diferencia } \\
\text { de } \mathbf{x}\end{array}$ & $\mathbf{p}$ & $\begin{array}{c}\text { 4mm } \\
\text { Diferencia } \\
\text { de } \mathbf{x}\end{array}$ & $\mathbf{p}$ \\
\hline $2 \mathrm{~mm}$ & $-15,0$ & 1,000 & & & & & & \\
$3 \mathrm{~mm}$ & $-69,3$ & 0,838 & $-53,8$ & 1,000 & & & & \\
$4 \mathrm{~mm}$ & $-121,5$ & $0,046^{*}$ & $-106,1$ & 0,114 & $-52,3$ & 1,000 & & 1,000 \\
$5 \mathrm{~mm}$ & $-86,6$ & 0,341 & $-71,1$ & 0,766 & $-17,3$ & 1,000 & 34,9 & \\
\hline
\end{tabular}

${ }^{*} \mathrm{p}<0,05$.

Tabla 6. Asociación entre marca y espesor de la resina con la resistencia a la compresión. $R^{*}=0,47$

\begin{tabular}{|c|c|c|}
\hline Variable & Coeficiente & p \\
\hline \multicolumn{3}{|l|}{ P60 } \\
\hline Tetric N Ceram & $-18,29$ & 0,266 \\
\hline SureFil & $-38,14$ & 0,022 \\
\hline \multicolumn{3}{|l|}{$1 \mathrm{~mm}$} \\
\hline $2 \mathrm{~mm}$ & $-89,01$ & 0,000 \\
\hline $3 \mathrm{~mm}$ & $-69,35$ & 0,002 \\
\hline $4 \mathrm{~mm}$ & $-140,72$ & 0,000 \\
\hline $5 \mathrm{~mm}$ & $-122,9$ & 0,000 \\
\hline
\end{tabular}

${ }^{*} \mathrm{R}=$ Coeficiente de determinación.

\section{DISCUSIÓN}

Los esfuerzos en desarrollo de materiales se han dirigido a solucionar inconvenientes relacionados con las propiedades de las resinas para el sector posterior, al aumentar la cantidad de material de relleno en la matriz de resina y al reducir el tamaño de la partícula de relleno. De la misma manera, se han estudiado la química, el método de polimerización y la unión entre el relleno y la matriz para eliminar los inconvenientes asociados con su color, propiedades físicas, químicas y mecánicas, pulido, cambios volumétricos de curado, manipulación y adhesión. ${ }^{4}$

La variación de métodos de prueba, la temperatura, tipos de resina, el lote del material, las soluciones en las que se almacenen, el tiempo de almacenamiento, el tamaño de la muestra, el diseño metodológico y el grado de conversión se encuentran, entre muchos factores, que influyen en los resultados. ${ }^{6}$

Es importante tener en cuenta que la profundidad y la extensión de las cavidades operatorias en boca varían. Según los resultados obtenidos y con las limitaciones de este estudio, proyectado a la actividad clínica, una restauración en resina que se realice en una cavidad superficial tendría (en cuanto a la resistencia a fuerzas compresivas) mayor éxito que una restauración en resina realizada en una cavidad más profunda. Sin embargo, en boca hay múltiples aspectos que influyen en el comportamiento de la restauración como la temperatura bucal, cambios dimensionales térmicos, estrato adhesivo, tipo de adhesivo, técnica de aplicación, tipo de luz e intensidad, uso de bases intermedias, ubicación en boca, tipo de preparación, tipo de dieta o hábitos, género y edad, entre otros.

Los valores de resistencia a la compresión que proveen los fabricantes de Filtek P60 (3M ESPE), la Tetric N Ceram (Ivoclar Vivadent) y los resultados de Díaz y colaboradores para la SureFil (Dentsply), ${ }^{4}$ no coinciden con los valores obtenidos en el presente estudio pues se observo que la resistencia (MPa) es menor. Esto se puede explicar por el reducido tamaño de la muestra $(\mathrm{n}=5){ }^{10}$

El estudio mostró una amplia variación que influyó en los resultados de la prueba, lo que se atribuye al tamaño de la muestra seleccionado. Se propone que para próximos estudios se seleccione un tamaño de muestra mayor.

Con las limitaciones de este estudio, las marcas de resina evaluadas no mostraron diferencias de importancia entre ellas, por lo tanto, no es posible afirmar que una resina sea superior a otra. Además, los tipos de materiales evaluados fueron fabricados para la misma indicación (obturaciones en el sector posterior) y para cumplir requisitos similares; al ser comparadas por espesor se obtuvo un resultado de significancia estadística en el espesor de $3 \mathrm{~mm}$. 
Aunque no se encontraron diferencias significativas entre las tres marcas de resinas ni en los diferentes espesores (salvo el de $3 \mathrm{~mm}$ ), fue algo mayor la fuerza requerida para fracturar las probetas de la Filtek P60 y los espesores más delgados mostraron mejor comportamiento ante las cargas compresivas empleadas. En la revisión de la literatura no se encontraron estudios de fuerzas compresivas en resinas relacionados con el espesor de las muestras; sin embargo, existen trabajos de investigación de este tipo de cargas en materiales de ingeniería como el concreto, en los cuales se obtuvieron resultados similares respecto al comportamiento de los espesores más delgados. ${ }^{11-13}$

Es de importancia aclarar que la sensibilidad para registrar el especimen del instrumento aplicador de carga de la máquina de ensayos no influye, ya que es igual para muestras delgadas o gruesas. Por lo tanto, el establecimiento del punto de fractura en cada probeta no depende de la máquina. Así, se elimina la injerencia de una variable externa en los resultados.

El rango de la fuerza masticatoria máxima funcional in vivo, según los diferentes autores a lo largo de los años, ha variado desde $122.5 \mathrm{~N}$ hasta $847 \mathrm{~N} .^{14,15}$ Van Steenberghe y colaboradores (1978) reportaron fuerzas distribuidas sobre los contactos de los dientes posteriores entre $122.5 \mathrm{~N}$ y $588 \mathrm{~N} .{ }^{13}$ Gibbs y colaboradores (1981) encontraron valores de $254.8 \mathrm{~N}$ durante la fase inmóvil de oclusión céntrica de la mandíbula. ${ }^{16}$ De otra parte, Hagberg (1986) encontró que las contracciones de los músculos masetero y temporal en la masticación producían fuerzas de elevación máximas de $245 \mathrm{~N}$ y $392 \mathrm{~N}$, respectivamente. ${ }^{17}$ Waltimo y colaboradores (1993) reportaron que los valores promedio de la fuerza de mordida en hombres era de $847 \mathrm{~N}$ y en mujeres $597 \mathrm{~N}$ en el sector posterior. ${ }^{14}$

En fuerzas masticatorias disfuncionales o eventos de bruxismo, Nishigawa y colaboradores (2001) obtuvieron valores promedio de $220.5 \mathrm{~N}$ a $414.5 \mathrm{~N}$, con amplitud máxima individual de 795.8 N. $^{18}$

En el presente estudio In vitro se obtuvieron valores de mínima y máxima resistencia de 1043.7N y $5040.6 \mathrm{~N}$, respectivamente. Al comparar estos valores con los valores in vivo de los estudios citados anteriormente, se observó claramente que en la fuerza de masticación se presentan valores significativamente menores, por lo tanto, se infiere que en boca no se presentarían cargas tan altas como las que se utilizaron en este estudio.
Se debe tener en cuenta que existen diferencias entre las fracturas que ocurren clínicamente y aquellas que hacen parte de una prueba In vitro. Las fuerzas creadas intraoralmente durante la masticación varían en magnitud, velocidad y dirección, 1 mientras que las fuerzas aplicadas a las muestras en este estudio fueron de velocidad y dirección constante e incremental hasta la falla. Con las limitaciones de este estudio, se podría señalar que las resinas para el sector posterior evaluadas tienen una buena resistencia compresiva para las cargas que se presentan durante el proceso de masticación.

Es importante destacar que la longevidad de la restauración en resina y su adecuado comportamiento no depende únicamente de cargas compresivas, también durante el proceso masticatorio se presentan fuerzas a tensión, flexión y cortantes simultáneamente. Por esto, se puede considerar que las muestras delgadas (las cuales presentaron mayor resistencia a la compresión) pueden comportarse adecuadamente frente a este tipo de fuerzas, más no frente a cargas a tensión y de corte que también se presentan en el ambiente oral.

Se propone que para estudios futuros se evalúe el comportamiento de acuerdo al espesor al aplicar otro tipo de fuerza. Así mismo, se sugiere emplear un diseño de estudio donde se puedan utilizar controles positivos (resinas de diferente indicación) y negativos (diferente material restaurador), que permitan una visión amplia de los resultados obtenidos, de manera que sea posible discutir más ampliamente y de diversas perspectivas y evaluar la fiabilidad de un producto al ser comparado con otro parecido o totalmente diferente para el mismo fin.

\section{Conclusiones}

- No hay diferencias estadísticamente significativas entre las marcas de resinas compuestas estudiadas.

- Se encontraron diferencias importantes asociadas al grosor de la muestra con mejores valores para las muestras más delgadas.

- El tamaño de muestra debe ampliarse en futuros trabajos.

- En próximos estudios, se sugiere utilizar controles positivos y negativos.

\section{Agradecimientos}

Los autores desean agradecer a los Drs. Gérman Gómez Mogollón, Luis Miguel Ramírez A., Juan Carlos Suárez y Harold Torres Pinzón por su valiosa colaboración en cuanto a la discusión y presentación de la información. 


\section{BIBLIOGRAFÍA}

1. Naranjo M, Ortiz P, Díaz M, Gómez M, Patiño MC. Resistencia a la fractura de dientes intactos y restaurados con resina sometidos a carga constante. Revista CES Odontología 2007; 20: 31 - 38

2. Anusavice KJ. Phillips Ciencia de los Materiales Dentales. 11 ed. Elsevier Science; 2008. p. 339 - 441

3. Aghazadeh Mohandesi J, Rafiee MA, Barzegaran V, Shafiei $\mathrm{F}$. Compressive fatigue behavior of dental restorative composites. Dent Mater 2007; 26: 827 - 837

4. Díaz JG, Gil JJ, Reyes LM, Bonilla A, Franco Y, Lara S, Peña D. Mechanical properties comparison for dental materials (ceromers and resins). VI COMEC Villa Clara, CUBA. Nov 2010

5. ANSI/ADA Specification No. 27. Resin-based composites J Am Dent Assoc 2003; 134: 510 - 512

6. Watanabe $\mathrm{H}$, Khera SC, Vargas MA, Qian F. Fracture toughness comparison of six resin composites. Dent Mater 2008; $24: 418-425$

7. Abe Y, Braem MJA, Lambrechts P, Inoue S, Takeuchi M, Van Meerbeek B. Fatigue behavior of packable composites. Biomaterials 2005; 26: 3405 - 3409

8. Norma Técnica Colombiana NTC 1810 - 2004. Materiales de Obturación, Restauración y Cementación con Base en Polímeros. ICONTEC 2009

9. Mitra SB, Wu D, Holmes NB. An application of nanotechnology in advanced dental materials. J Am Dent Assoc 2003; 134: 1382 - 1390

10. Kim JK, Yi ST, Kim JJ. Effect of specimen sizes on flexural compressive strength of concrete. ACI Structural Journal 2001; 98: 416 - 424

11. French CW. Mokhtarzadeh A. High strength concrete: effects of material, curing and test procedures on shortterm compressive strength. PCI Journal 1993; 38: 76 - 87

12. Vandegrift D, Schindler AK. The Effect of Test Cylinder Size on the Compressive Strength of Sulfur Capped Concrete Specimens. Highway Research Center and Department of Civil Engineering at Auburn University [en línea] 2006. URL disponible en: http://www.eng.auburn. edu/files/file806.pdf

13. Van Steenberghe D, De Vries IH. The development of a maximal clenching force between two antagonistic teeth. J Periodontal Res 1978; 13: 91 - 97

14. Waltimo A, Konotten M. A novel bite force recorder and maximal isometric bite force values for healthy young adults. Scand J Dent Res 1993: $101-171$

15. Christensen LV, McKay DC. Kinematic and kinetic observations on ballistic depression and elevation of the human mandible. J Oral Rehabil 2000; 27: 494 - 507

16. Gibbs $\mathrm{CH}$, Mahan $\mathrm{PE}$, Lundeen $\mathrm{HC}$, Brehnan $\mathrm{K}$, Walsh EK, Sinkewiz SL, Ginsberg SB. Occlusal forces during chewing - influences of biting strength and food consistency. J Prosthet Dent 1981; 46: 561 - 567

17. Hagberg C. The amplitud distribution of electromyographic activity of masticatory muscles during unilateral chewing. J Oral Rehabil 1986; 13: 567 - 574

18. Nishigawa K, Bando E, Nakano M. Quantitative study of bite force during sleep-associated bruxism. J Oral Rehabil 2001; $28: 485-491$
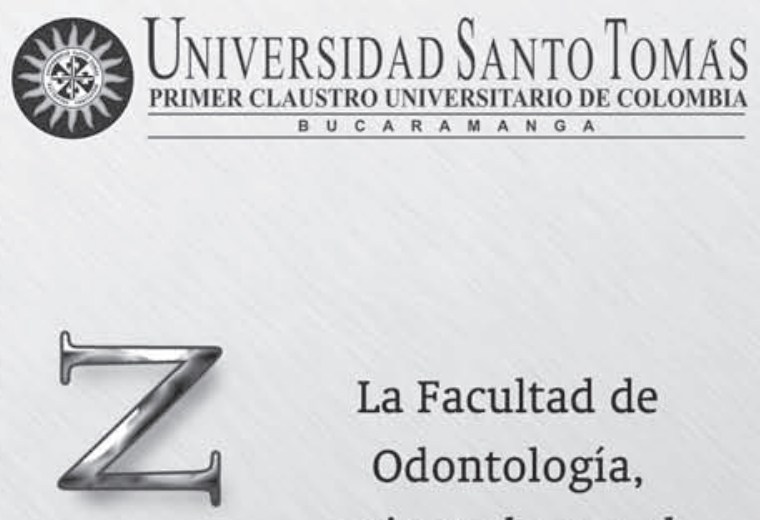

La Facultad de

Odontología, orientada por el pensamiento de

Santo Tomás,

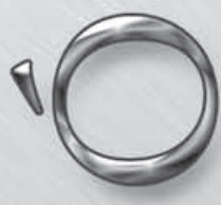

pretende formar

Odontólogos

integrales $\mathrm{y} / \mathrm{o}$

especialistas a través

de parámetros

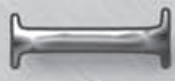

humanísticos, éticos, científicos, biotecnológicos,

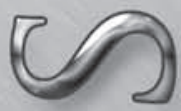
investigativos $\mathrm{y}$ sociales, como recurso humano capaz de

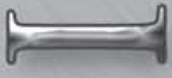
intervenir con éxito en el proceso dinámico de la salud y la enfermedad, en el

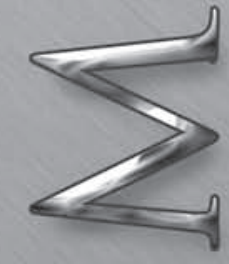
individuo, la familia y la comunidad. 